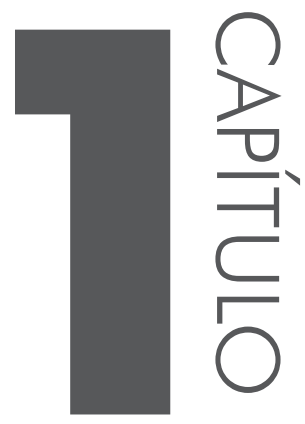

\title{
ESTUDOS DE SINGULARIDADES NO FLUXO DE CARGA CONTINUADO
}

Alfredo Bonini Neto

Universidade Estadual Paulista - Unesp, câmpus de Tupã-SP, Departamento de Engenharia de Biossistemas. bonini@tupa.unesp.br

Elisabete de Mello Magalhães

Universidade Estadual Paulista - Unesp, câmpus de Ilha Solteira-SP, Departamento de Engenharia Elétrica. elisabete.magalhaes@yahoo.com.br
Dilson Amancio Alves Universidade Estadual Paulista - Unesp, câmpus de Ilha Solteira-SP, Departamento de Engenharia Elétrica. dalves@dee.feis.unesp.br

\section{RESUMO}

Nos últimos anos, a preocupação com o assunto estabilidade de tensão vem ganhando destaque mundial quando se refere à indústria do setor energético e isso se deve ao fato de esta questão está relacionada com a operação e o planejamento de sistemas elétricos de potência. Fatores como o crescente aumento da demanda de energia e a transferência de elevadas quantidades de potência para atender ao consumo, aliados às exigências econômicas e ambien- 
tais têm levado os sistemas a operarem em condições estressantes (próximos de seus limites), ou seja, com pequenas margens de segurança, representando uma ameaça à sua condição de operação estável. A combinação desses fatores pode ser desastrosa, pois torna os sistemas elétricos de potência vulneráveis, expostos ao risco de uma situação de instabilidade. Na literatura técnica, um dos estudos para análise de estabilidade e instabilidade de tensão está relacionado à curva P-V (potência versus magnitude da tensão) e ao ponto na curva que separa a operação estável da instável, denominado ponto de máximo carregamento (PMC). O PMC pode ser consequente de uma bifurcação de sela-nó (BSN), relacionada com o limite de capacidade de transmissão num sistema elétrico, no qual a matriz Jacobiana é singular, ou de uma bifurcação estática induzida por limites (BIL), relacionada com o limite de potência reativa do gerador, no qual a matriz não é singular. Nesse sentido, apresenta-se, no início deste capítulo, uma análise de algumas das formulações dos métodos de fluxo de carga continuado (FCC), abordados pela literatura. O estudo foi desenvolvido de maneira a analisar as modificações que as técnicas de parametrização acarretam na matriz Jacobiana convencional $(\mathbf{J})$, de forma a eliminar os problemas de singularidade de $\mathbf{J}$ no PMC e nos pontos de singularidade da matriz de cada método. Este capítulo apresenta também a trajetória dos valores dos determinantes das matrizes Jacobianas modificadas $\left(\mathrm{I}_{\mathrm{m}} \mid\right)$ dos métodos já abordados. Uma análise gráfica também é mostrada para uma melhor compreensão. Os métodos estudados tratam de parametrização local e global, utilizando ou a perda total de potência ativa $(\mathrm{Pa})$; ou o coeficiente angular de retas localizadas no plano formado pela soma das magnitudes de tensão nodais $\left(\Sigma \mathrm{V}_{k}\right)$, ou ângulos nodais $\left(\Sigma_{k}\right)$, e fator de carregamento; ou as equações de quadráticas, situadas no plano formado por $\mathrm{Pa}$ e fator de carregamento. Os resultados são obtidos para os sistemas do IEEE-14 e 300 barras. Um estudo no PMC é efetuado a fim de verificar se o ponto corresponde a uma BSN ou a uma BIL.

Palavras-chaves: Estabilidade de tensão, Ponto de máximo carregamento, Bifurcação, Fluxo de carga continuado, Método da continuação.

\section{INTRODUC̣ÃO}

Atualmente, com o aumento crescente da demanda de energia elétrica, os estudos dos métodos de fluxo de carga continuado têm aumentado de forma significativa em todo o globo. A importância desses métodos se deve ao fato de eles possibilitarem a obtenção do PMC com precisão preestabelecida e sem problemas numéricos, o traçado completo das curvas $\mathrm{P}-\mathrm{V}$ e a determinação da margem segura de carregamento. Vários métodos foram desenvolvidos visando obter toda a curva $\mathrm{P}-\mathrm{V}$ e eliminar os problemas numéricos relacionados à matriz J (AJJARAPU; 
CHRISTY, 1992), (BONINI NETO; ALVES, 2010 a), (BONINI NETO; ALVES, 2014 $4_{\mathrm{a}}$, (BONINI NETO; ALVES, 2014 ), (GARBELINI et al., 2007), (BONINI NETO; ALVES, 2010 ) e (MAGALHÃES et al., 2013). Em Ajjarapu e Christy (1992), foi proposto um método da continuação, utilizando uma técnica de parametrização, denominada de parametrização local. Nesse método, o parâmetro de continuação inicialmente escolhido é o fator de carregamento do sistema $(\lambda)$. Posteriormente, próximo ao PMC, efetua-se a troca, sendo que a variável escolhida como novo parâmetro é aquela que apresentar a maior variação no vetor tangente. Em geral, ou magnitude da tensão nodal $\left(\mathrm{V}_{\mathrm{k}}\right)$ ou ângulo da tensão nodal $\left(\theta_{\mathrm{k}}\right)$ de uma barra k qualquer é escolhida como novo parâmetro da continuação. Em Bonini Neto e Alves (2010 a), também foi apresentada uma técnica de parametrização local para o método da continuação envolvendo equações de retas no plano cartesiano, fator de carregamento $(\lambda)$ e magnitude da tensão nodal $\left(V_{k}\right), \lambda-V_{k}$. A singularidade da matriz J é eliminada pela equação de uma reta passando por esse plano. Com isso, ao se incrementar o coeficiente angular dessa reta, obtêm-se as sucessivas soluções do fluxo de carga. Uma ideia similar foi apresentada em Bonini Neto e Alves (2014 ) e Bonini Neto e Alves (2014 ), a diferença é que o plano para a obtenção das curvas $\mathrm{P}-\mathrm{V}$ é formado pelo fator de carregamento $(\lambda)$ e pelo ângulo da tensão nodal $\left(\theta_{\mathrm{k}}\right), \lambda-\theta_{\mathrm{k}}$. Em Garbelini et al. (2007), apresentou-se uma técnica global de parametrização geométrica que possibilita o traçado completo das curvas P-V sem os problemas de mau condicionamento. Na técnica, que associa a robustez com a simplicidade, a singularidade da matriz J é eliminada pela adição da equação de uma reta que passa por um ponto no plano formado pelas variáveis fator de carregamento $(\lambda)$ e perda total de potência ativa $(\mathrm{Pa})$. Em Bonini Neto e Alves $\left(2010_{b}\right)$, apresenta-se uma eficiente técnica global de parametrização geométrica para o fluxo de carga continuado, na qual a singularidade da matriz $\mathbf{J}$ é eliminada pela adição de uma equação da reta que passa através dos pontos no plano determinado pelas variáveis fator de carregamento $(\lambda)$ e soma de todas as magnitudes das tensões nodais $\left(\Sigma \mathrm{V}_{\mathrm{k}}\right)$, plano $\lambda-\Sigma \mathrm{V}_{\mathrm{k}}$, ou de todos os ângulos nodais $\left(\Sigma \theta_{\mathrm{k}}\right)$, plano $\lambda-\Sigma \theta_{\mathrm{k}}$. E, por fim, em Magalhães et al. (2013), utiliza-se a equação de uma quadrática no plano formado pelas variáveis $\lambda-P a$. As vantagens e particularidades de cada uma dessas técnicas podem ser verificadas nas respectivas referências. Na literatura, existem diversas outras propostas para o método de fluxo de carga continuado (CHIANG et al., 1999), (SEYDEL, 1994), (CHIANG et al., 1995), (ALVES et al., 2000), (ZHAO; ZHANG, 2006), (MAGALHÃES et al., 2012), (LI; CHIANG, 2008) e (MORI; SEKI, 2009).

Uma das alternativas para verificar a singularidade da matriz $\mathbf{J}$ é inverter o sinal do determinante no PMC, ou seja, o determinante é nulo exatamente no 
PMC, podendo ser verificado em sistemas de pequeno porte por meio do gráfico dos valores numéricos do determinante em função do carregamento $\lambda$. A dimensão da matriz quadrada $J$ é da ordem de $\left(2 \mathrm{n}_{\mathrm{PQ}}+\mathrm{n}_{\mathrm{PV}}\right)$, sendo que $\mathrm{n}_{\mathrm{PQ}}$ e $\mathrm{n}_{\mathrm{PV}}$ correspondem ao número de barras PQ e PV, respectivamente. Então, por exemplo, para um sistema como o IEEE-14 barras, que é representado por 9 barras de carga, 5 barras de geração e 20 linhas de transmissão (LT), a matriz J convencional é da ordem de $23 \times 23(2 \times 9+5)$. Sistemas de grande porte como o IEEE-300 barras podem apresentar matrizes Jacobianas da ordem $530 \times 530$, acarretando em valores muito altos para o determinante, maiores do que $1 \mathrm{e}+309$. Determinantes dessa ordem são representados no MATLAB pelo símbolo inf ( $\infty$, infinito). Como alternativa para visualizar graficamente os seus valores, efetua-se a sua redução através da divisão dos elementos da matriz por 10.

Neste trabalho, é apresentada uma modelagem matemática dos métodos aqui citados, ou seja, apresenta-se uma análise da matriz Jacobiana dos métodos de fluxo de carga continuado, uma vez que, para cada método, ocorrem modificações na matriz Jacobiana $(\mathrm{J})$ convencional em consequência das reparametrizações necessárias para se eliminar os problemas de singularidade de J no PMC. Também são apresentados os valores dos determinantes para se verificarem as possíveis ocorrências de singularidades da matriz $\mathrm{J}_{\mathrm{m}}$ de cada método estudado, bem como o número de iterações necessárias para a obtenção dos pontos representando a curva P-V. Outro aspecto importante mostrado neste trabalho são os valores dos determinantes de cada ponto pertencente à geometria da trajetória de soluções do problema de fluxo de carga parametrizado, explicitando que a impossibilidade de obtenção do PMC pelo método de Newton convencional se deve a problemas numéricos (singularidade). O mesmo já não ocorre para a matriz Jacobiana modificada $\mathbf{J}_{\mathrm{m}}$ quando da escolha adequada do parâmetro. Um estudo no PMC é efetuado com o intuito de verificar se o ponto corresponde a uma BSN ou a uma BIL.

\section{MATRIZ JACOBIANA DO FLUXO DE CARGA CONTINUADO}

As equações do fluxo de carga são:

$$
\begin{aligned}
& \mathrm{G}(\theta, \mathrm{V})=0, \text { ou } \\
& \Delta \mathrm{P}=\mathrm{P}^{\mathrm{esp}}-\mathrm{P}(\theta, \mathrm{V})=0 \\
& \Delta \mathrm{Q}=\mathrm{Q}^{\mathrm{esp}}-\mathrm{Q}(\theta, \mathrm{V})=0
\end{aligned}
$$

sendo: 


$$
\begin{aligned}
& \mathrm{P}_{\mathrm{k}}(\theta, \mathrm{V})=V_{k} \sum_{m \in \kappa} V_{m}\left(G_{k m} \cos \theta_{k m}+B_{k m} \sin \theta_{k m}\right) \\
& \mathrm{Q}_{\mathrm{k}}(\theta, \mathrm{V})=V_{k} \sum_{m \in \kappa} V_{m}\left(G_{k m} \sin \theta_{k m}-B_{k m} \cos \theta_{k m}\right)
\end{aligned}
$$

V e $\theta$ são os respectivos vetores de magnitude de tensão nodal e ângulo de fase; $\mathbf{P}^{\text {esp }}$ é o vetor da diferença entre os vetores de potência ativa, gerada $\left(\mathbf{P}_{\mathrm{g}}\right.$ esp $)$ e consumida $\left(\mathbf{P}_{\mathbf{c}}^{\text {esp }}\right)$, especificada para as barras de carga $(P Q)$ e geração $(P V)$; e $Q^{\text {esp }}$ é o vetor de potência reativa consumida especificada para as barras $P Q$.

A Equação (1) assume que o carregamento da rede é proporcional ao do caso base e considera o fator de potência constante. $\mathrm{P}^{\text {esp }}$ e $\mathrm{Q}^{\text {esp }}$ também podem ser definidos como sendo iguais a $\left(\mathbf{k}_{\mathrm{Pg}} \mathbf{P}_{\mathrm{g}}^{\text {esp }}+\mathbf{k}_{\mathrm{Pc}_{\mathrm{c}}} \mathbf{P}_{\mathrm{c}}^{\text {esp }}\right)$ e $\mathbf{k}_{\mathrm{Qc}} \mathbf{Q}_{\mathrm{c}}$ esp , respectivamente. Os vetores $\mathbf{k}_{\mathrm{Pg}}, \mathbf{k}_{\mathrm{Pc}_{\mathrm{c}}}$ e $\mathbf{k}_{\mathrm{Qc}}$ são parâmetros fixos usados para caracterizar um cenário específico de carga.

A resolução do sistema de Equações (1) pelo método de Newton é feita por meio da linearização da função $\mathrm{G}(\mathrm{x})$, em que $\mathrm{G}(\mathrm{x})=[\Delta \mathrm{P}, \Delta \mathrm{Q}]^{\prime}=\left[\mathrm{G}_{1}, \mathrm{G}_{2}, \ldots\right]^{\prime}$ e $\mathbf{x}=[\theta, V]$, para $\mathbf{x}=\mathbf{x}^{\mathrm{i}}$, considerando os dois primeiros termos da série de Taylor:

$$
G\left(x^{i}+\Delta x^{i}\right) \cong G\left(x^{i}\right)+J\left(x^{i}\right) \Delta x^{i}
$$

sendo a matriz jacobiana J dada por:

$$
J=\frac{\partial G}{\partial x}=\left[\begin{array}{cccc}
\frac{\partial G_{1}}{\partial x_{1}} & \frac{\partial G_{1}}{\partial x_{2}} & \cdots & \frac{\partial G_{1}}{\partial x_{n}} \\
\frac{\partial G_{2}}{\partial x_{1}} & \frac{\partial G_{2}}{\partial x_{2}} & \cdots & \frac{\partial G_{2}}{\partial x_{n}} \\
\cdots & \cdots & \cdots & \cdots \\
\frac{\partial G_{n}}{\partial x_{1}} & \frac{\partial G_{n}}{\partial x_{2}} & \cdots & \frac{\partial G_{n}}{\partial x_{n}}
\end{array}\right]
$$

O vetor de correção $\Delta x$ é calculado, impondo-se que:

$$
\mathrm{G}\left(\mathrm{x}^{\mathrm{i}}\right)+\mathrm{J}\left(\mathrm{x}^{\mathrm{i}}\right) \Delta \mathrm{x}^{\mathrm{i}}=0
$$

que é a maneira linearizada de se resolver o problema $G\left(x^{i}+\Delta x^{i}\right)=0$. No caso em que $n=2$, a Equação (3) assume a forma:

$$
\begin{aligned}
& \mathrm{G}_{1}\left(\mathrm{x}_{1}, \mathrm{x}_{2}\right) \cong \mathrm{G}_{1}\left(\mathrm{x}_{1}{ }^{\mathrm{i}}, \mathrm{x}_{2}{ }^{\mathrm{i}}\right)+\left.\frac{\partial \mathrm{G}_{1}}{\partial \mathrm{x}_{1}}\right|_{\mathrm{i}} \Delta \mathrm{x}_{1}{ }^{(\mathrm{i})}+\left.\frac{\partial \mathrm{G}_{1}}{\partial \mathrm{x}_{2}}\right|_{\mathrm{i}} \Delta \mathrm{x}_{2}{ }^{(\mathrm{i})} \\
& \mathrm{G}_{2}\left(\mathrm{x}_{1}, \mathrm{x}_{2}\right) \cong \mathrm{G}_{2}\left(\mathrm{x}_{1}{ }^{\mathrm{i}}, \mathrm{x}_{2}{ }^{\mathrm{i}}\right)+\left.\frac{\partial \mathrm{G}_{2}}{\partial \mathrm{x}_{1}}\right|_{\mathrm{i}} \Delta \mathrm{x}_{1}{ }^{(\mathrm{i})}+\left.\frac{\partial \mathrm{G}_{2}}{\partial \mathrm{x}_{2}}\right|_{\mathrm{i}} \Delta \mathrm{x}_{2}{ }^{(\mathrm{i})}
\end{aligned}
$$

e, na forma matricial, representa: 


$$
\left[\begin{array}{l}
\mathrm{G}_{1}\left(\mathrm{x}_{1}{ }^{\mathrm{i}}, \mathrm{x}_{2}{ }^{\mathrm{i}}\right) \\
\mathrm{G}_{2}\left(\mathrm{x}_{1}{ }^{\mathrm{i}}, \mathrm{x}_{2}{ }^{\mathrm{i}}\right)
\end{array}\right]+\left[\begin{array}{ll}
\frac{\partial \mathrm{G}_{1}}{\partial \mathrm{x}_{1}} & \frac{\partial \mathrm{G}_{1}}{\partial \mathrm{x}_{2}} \\
\frac{\partial \mathrm{G}_{2}}{\partial \mathrm{x}_{1}} & \frac{\partial \mathrm{G}_{2}}{\partial \mathrm{x}_{2}}
\end{array}\right]^{(i)} \cdot\left[\begin{array}{c}
\Delta \mathrm{x}_{1}{ }^{(\mathrm{i})} \\
\Delta \mathrm{x}_{2}{ }^{(\mathrm{i})}
\end{array}\right]=\left[\begin{array}{l}
0 \\
0
\end{array}\right]
$$

A nova solução $\mathbf{x}^{(i+1)}$ é:

$$
x^{i+1}=x^{i}+\Delta x^{i}
$$

e:

$$
\Delta \mathrm{x}^{\mathrm{i}}=-\left[\mathrm{J}\left(\mathrm{x}^{\mathrm{i}}\right)\right]^{-1} \mathrm{G}\left(\mathrm{x}^{\mathrm{i}}\right)
$$

Para o fluxo de carga continuado, o sistema de Equações (1) passa a ter uma variável a mais, ou seja, adiciona-se uma variável $\lambda$ que representa o fator de carregamento do sistema, tornando-se:

$$
\begin{aligned}
& \mathrm{G}(\theta, \mathrm{V}, \lambda)=0, \text { ou } \\
& \Delta \mathrm{P}=\lambda \mathrm{P}^{\mathrm{esp}}-\mathrm{P}(\theta, \mathrm{V})=0 \\
& \Delta \mathrm{Q}=\lambda \mathrm{Q}^{\mathrm{esp}}-\mathrm{Q}(\theta, \mathrm{V})=0
\end{aligned}
$$

O traçado da curva P-V pode ser feito por sucessivas soluções de (10), utilizando um FC. O PMC é obtido através da adoção de $\lambda$ como um parâmetro. Seu valor é aumentado gradualmente a partir do caso base $(\lambda=1)$ até um valor para o qual nenhuma solução possa ser obtida, ou seja, para o qual o processo iterativo do fluxo de potência não convirja ou divirja. Em geral, nesse ponto, haverá um controle de passo consistindo de uma simples redução no incremento (tamanho do passo) de $\lambda$, e a nova solução é obtida a partir da última solução convergente. O PMC é considerado o último ponto convergido após sucessivas repetições desse procedimento. No entanto, a divergência do FC é uma consequência da singularidade da matriz Jacobiana (J) de (10) no PMC, e, portanto, seu valor não pode ser determinado com precisão. A fim de superar as dificuldades numéricas resultantes da singularidade de $\mathbf{J}$ e permitir a determinação do PMC, foram propostas diferentes parametrizações para o método da continuação (AJJARAPU; CHRISTY, 1992), (BONINI NETO; ALVES, 2010) ), (BONINI NETO; ALVES, 2014 $)$, (BONINI NETO; ALVES, 2014 b $_{\mathrm{b}}$, (GARBELINI et al, 2007), (BONINI NETO; ALVES, 2010 ), (MAGALHÃES et al., 2012), (MAGALHÃES et al., 2013), (CHIANG et al., 1999), (SEYDEL, 1994), (CHIANG et al., 1995), (ALVES et al., 2000), (ZHAO; ZHANG, 2006), (LI; CHIANG, 2008) e (MORI; SEKI, 2009). Como o sistema apresenta uma variável a mais do que o número de equações, é 
necessário acrescentar mais uma equação. Logo, a partir da Equação (10), cada método apresenta a sua particularidade.

\section{ANÁLISE DA MATRIZ JACOBIANA MODIFICADA DO FLUXO DE CARGA CONTINUADO}

A equação $E(\theta, V, \lambda)$ foi acrescentada ao sistema de Equações (10) no método proposto em Ajjarapu e Christy (1992), passando a ser:

$$
\left\{\begin{array}{l}
\Delta \mathrm{P}=\lambda \mathrm{P}^{\mathrm{esp}}-\mathrm{P}(\theta, \mathrm{V})=0 \\
\Delta \mathrm{Q}=\lambda \mathrm{Q}^{\mathrm{esp}}-\mathrm{Q}(\theta, \mathrm{V})=0 \\
\mathrm{E}(\theta, \mathrm{V}, \lambda)=\left(\lambda-\lambda^{0}\right) \text { ou }\left(\mathrm{V}_{\mathrm{k}}-\mathrm{V}_{\mathrm{k}}^{0}\right) \text { ou }\left(\theta_{\mathrm{k}}-\theta_{\mathrm{k}}^{0}\right)=0
\end{array}\right.
$$

Linearizando pelos dois primeiros termos da série de Taylor:

$$
\begin{aligned}
& \Delta \mathrm{P}+\frac{\partial \Delta \mathrm{P}}{\partial \theta} \Delta \theta+\frac{\partial \Delta \mathrm{P}}{\partial \mathrm{V}} \Delta \mathrm{V}+\frac{\partial \Delta \mathrm{P}}{\partial \lambda} \Delta \lambda=0 \\
& \Delta \mathrm{Q}+\frac{\partial \Delta \mathrm{Q}}{\partial \theta} \Delta \theta+\frac{\partial \Delta \mathrm{Q}}{\partial \mathrm{V}} \Delta \mathrm{V}+\frac{\partial \Delta \mathrm{Q}}{\partial \lambda} \Delta \lambda=0 \\
& \mathrm{E}+\frac{\partial \mathrm{E}}{\partial \theta} \Delta \theta+\frac{\partial \mathrm{E}}{\partial \mathrm{V}} \Delta \mathrm{V}+\frac{\partial \mathrm{E}}{\partial \lambda} \Delta \lambda=0
\end{aligned}
$$

que, na forma matricial, torna-se:

$$
\begin{gathered}
{\left[\begin{array}{c}
\Delta \mathrm{P} \\
\Delta \mathrm{Q} \\
\mathrm{E}
\end{array}\right]+\left[\begin{array}{ccc}
\frac{\partial \Delta \mathrm{P}}{\partial \theta} & \frac{\partial \Delta \mathrm{P}}{\partial \mathrm{V}} & \frac{\partial \Delta \mathrm{P}}{\partial \lambda} \\
\frac{\partial \Delta \mathrm{Q}}{\partial \theta} & \frac{\partial \Delta \mathrm{Q}}{\partial \mathrm{V}} & \frac{\partial \Delta \mathrm{Q}}{\partial \lambda} \\
\frac{\partial \mathrm{E}}{\partial \theta} & \frac{\partial \mathrm{E}}{\partial \mathrm{V}} & \frac{\partial \mathrm{E}}{\partial »}
\end{array}\right]\left[\begin{array}{c}
\Delta \theta \\
\Delta \mathrm{V} \\
\Delta \lambda
\end{array}\right]=0} \\
{\left[\begin{array}{c}
\Delta \mathrm{P} \\
\Delta \mathrm{Q} \\
\mathrm{E}
\end{array}\right]+\left[\begin{array}{ccc}
-\frac{\partial \mathrm{P}}{\partial \theta} & -\frac{\partial \mathrm{P}}{\partial \mathrm{V}} & \mathrm{P}^{\text {esp }} \\
-\frac{\partial \mathrm{Q}}{\partial \theta} & -\frac{\partial \mathrm{Q}}{\partial \mathrm{V}} & \mathrm{Q}^{\text {esp }} \\
\frac{\partial \mathrm{E}}{\partial \theta} & \frac{\partial \mathrm{E}}{\partial \mathrm{V}} & \frac{\partial \mathrm{E}}{\partial \lambda}
\end{array}\right]\left[\begin{array}{c}
\Delta \theta \\
\Delta \mathrm{V} \\
\Delta \lambda
\end{array}\right]=0}
\end{gathered}
$$

Caso o parâmetro for a variável $\lambda$, Figura $1(\mathrm{a})$, então se utiliza $\mathrm{E}(\theta, \mathrm{V}, \lambda)=\left(\lambda-\lambda^{0}\right)=0, \mathrm{e}$, nesse caso: 


$$
\left[\begin{array}{c}
\Delta \mathrm{P} \\
\Delta \mathrm{Q} \\
\mathrm{E}
\end{array}\right]+\left[\begin{array}{ccc}
-\frac{\partial \mathrm{P}}{\partial \theta} & -\frac{\partial \mathrm{P}}{\partial \mathrm{V}} & \mathrm{P}^{\text {esp }} \\
-\frac{\partial \mathrm{Q}}{\partial \theta} & -\frac{\partial \mathrm{Q}}{\partial \mathrm{V}} & \mathrm{Q}^{\mathrm{esp}} \\
0 & 0 & 1
\end{array}\right]\left[\begin{array}{c}
\Delta \theta \\
\Delta \mathrm{V} \\
\Delta \lambda
\end{array}\right]=0
$$

ou:

$$
-\left[\begin{array}{ccc}
-\mathbf{J} & & \mathrm{P}^{\text {esp }} \\
0 & 0 & 1
\end{array}\right]\left[\begin{array}{c}
\Delta \mathbf{x} \\
\Delta \lambda
\end{array}\right]=-\mathbf{J}_{\mathbf{m}}\left[\begin{array}{c}
\Delta \mathbf{x} \\
\Delta \lambda
\end{array}\right]=\left[\begin{array}{c}
\Delta \mathbf{G} \\
\mathrm{E}
\end{array}\right]
$$

em que $\mathbf{J}_{\mathrm{m}}$ é dada por:

$$
\left[\begin{array}{ccc}
-\mathbf{J} & \mathrm{P}^{\mathrm{esp}} \\
0 & 0 & \mathrm{Q}^{\text {esp }} \\
0 & 0 & 1
\end{array}\right]
$$

ou por:

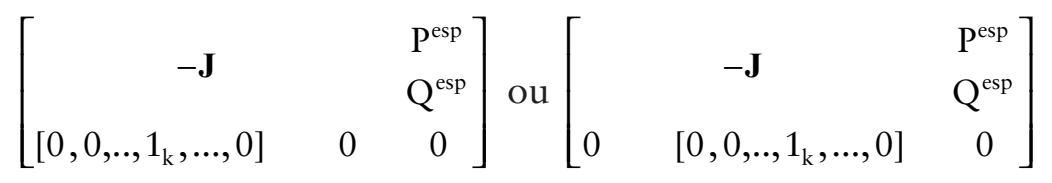

caso a variável escolhida como parâmetro seja $\theta_{\mathrm{k}}\left(\mathrm{E}(\theta, \mathrm{V}, \lambda)=\left(\theta_{\mathrm{k}}-\theta_{\mathrm{k}}^{0}\right)=0\right)$ ou $\mathrm{V}_{\mathrm{k}}$ $\left(\mathrm{E}(\theta, \mathrm{V}, \lambda)=\left(\mathrm{V}_{\mathrm{k}}-\mathrm{V}_{\mathrm{k}}^{0}\right)=0\right)$, Figura $1(\mathrm{~b})$.
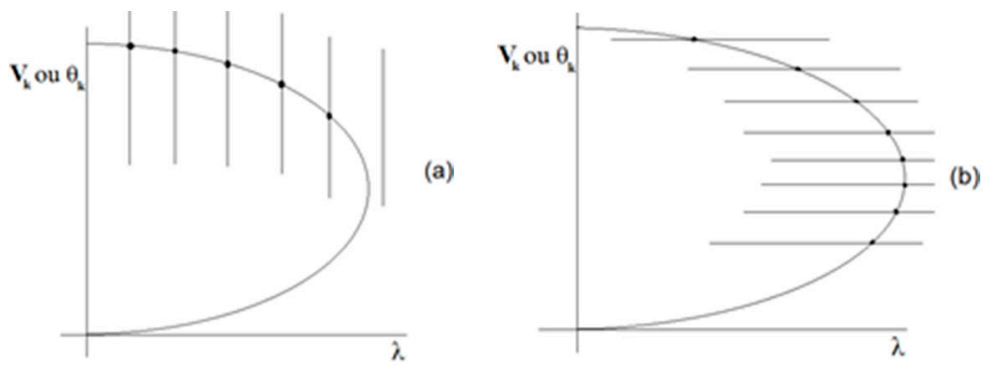

Figura 1 Método da parametrização local: (a) $\lambda$ como parâmetro, (b) $V_{k}$ ou $\theta_{k}$ como parâmetro.

No método apresentado em Bonini Neto e Alves (2010a), acrescentou-se ao sistema de Equações (10) a equação de uma reta W:

$$
\left\{\begin{array}{l}
\Delta \mathrm{P}=\lambda \mathrm{P}^{\mathrm{esp}}-\mathrm{P}(\theta, \mathrm{V})=0 \\
\Delta \mathrm{Q}=\lambda \mathrm{Q}^{\mathrm{esp}}-\mathrm{Q}(\theta, \mathrm{V})=0 \\
\mathrm{~W}(\theta, \mathrm{V}, \lambda, \pm)= \pm\left(\lambda-\lambda^{0}\right)-\left(\mathrm{V}_{\mathrm{k}}-\mathrm{V}_{\mathrm{k}}^{0}\right)=0
\end{array}\right.
$$


sendo $\alpha$ o coeficiente angular da reta, e $1^{0}$ e $V_{k}^{0}$ os pontos escolhidos no plano representando o centro do feixe de retas inicial, Figura 2(a), os quais podem ser alterados.

Linearizando a Equação (19) pelos dois primeiros termos da série de Taylor, tem-se:

$$
\begin{aligned}
& \Delta \mathrm{P}+\frac{\partial \Delta \mathrm{P}}{\partial \theta} \Delta \theta+\frac{\partial \Delta \mathrm{P}}{\partial \mathrm{V}} \Delta \mathrm{V}+\frac{\partial \Delta \mathrm{P}}{\partial \lambda} \Delta \lambda=0 \\
& \Delta \mathrm{Q}+\frac{\partial \Delta \mathrm{Q}}{\partial \theta} \Delta \theta+\frac{\partial \Delta \mathrm{Q}}{\partial \mathrm{V}} \Delta \mathrm{V}+\frac{\partial \Delta \mathrm{Q}}{\partial \lambda} \Delta \lambda=0 \\
& \mathrm{~W}+\frac{\partial \mathrm{W}}{\partial \theta} \Delta \theta+\frac{\partial \mathrm{W}}{\partial \mathrm{V}} \Delta \mathrm{V}+\frac{\partial \mathrm{W}}{\partial \lambda} \Delta \lambda=0
\end{aligned}
$$

que, na forma matricial, torna-se:

$$
\left[\begin{array}{c}
\Delta \mathrm{P} \\
\Delta \mathrm{Q} \\
\mathrm{W}
\end{array}\right]+\left[\begin{array}{ccc}
\frac{\partial \Delta \mathrm{P}}{\partial \theta} & \frac{\partial \Delta \mathrm{P}}{\partial \mathrm{V}} & \frac{\partial \Delta \mathrm{P}}{\partial \lambda} \\
\frac{\partial \Delta \mathrm{Q}}{\partial \theta} & \frac{\partial \Delta \mathrm{Q}}{\partial \mathrm{V}} & \frac{\partial \Delta \mathrm{Q}}{\partial \lambda} \\
\frac{\partial \mathrm{W}}{\partial \theta} & \frac{\partial \mathrm{W}}{\partial \mathrm{V}} & \frac{\partial \mathrm{W}}{\partial \lambda}
\end{array}\right]\left[\begin{array}{c}
\Delta \theta \\
\Delta \mathrm{V} \\
\Delta \lambda
\end{array}\right]=0
$$

ou:

$$
\left[\begin{array}{c}
\Delta \mathrm{P} \\
\Delta \mathrm{Q} \\
\mathrm{W}
\end{array}\right]+\left[\begin{array}{ccc}
-\frac{\partial \mathrm{P}}{\partial \theta} & -\frac{\partial \mathrm{P}}{\partial \mathrm{V}} & \mathrm{P}^{\text {esp }} \\
-\frac{\partial \mathrm{Q}}{\partial \theta} & -\frac{\partial \mathrm{Q}}{\partial \mathrm{V}} & \mathrm{Q}^{\text {esp }} \\
0 & -\left[0,0, . ., 1_{\mathrm{k}}, \ldots, 0\right] & \pm
\end{array}\right]\left[\begin{array}{c}
\Delta \theta \\
\Delta \mathrm{V} \\
\Delta \lambda
\end{array}\right]=0
$$

ou:

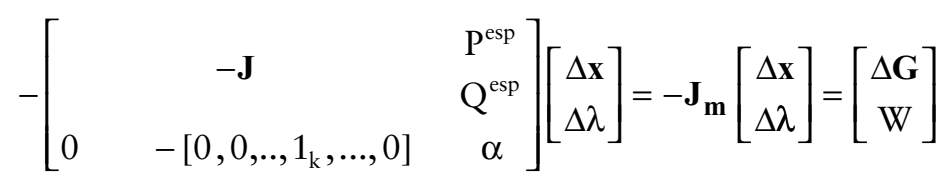

sendo $\mathrm{J}_{\mathrm{m}}$ dada por:

$$
\mathbf{J}_{\mathbf{m}}=\left[\begin{array}{ccc}
-\mathbf{J} & \mathrm{P}^{\operatorname{esp}} \\
-\left[0,0, . ., 1_{\mathrm{k}}, \ldots, 0\right] & 0 & -
\end{array}\right]
$$

Formulações similares foram obtidas em Bonini Neto e Alves $\left(2014_{a}\right)$ e Bonini Neto e Alves (2014 $)$ ), a diferença é que o plano utilizado para obtenção da 
curva P-V é o $\lambda-\theta_{\mathrm{k}}$, Figura 2(a). Nesse caso, acrescentou-se ao sistema de Equações (10) a equação da seguinte reta $\mathrm{R}$ :

$$
\mathrm{R}(\theta, \mathrm{V}, \lambda, \alpha)=\alpha\left(\lambda-\lambda^{0}\right)-\left(\theta_{\mathrm{k}}-\theta_{\mathrm{k}}^{0}\right)=0
$$

obtendo-se assim a matriz $\mathrm{J}_{\mathrm{m}}$ :

$$
\mathbf{J}_{\mathbf{m}}=\left[\begin{array}{ccc}
-\mathbf{J} & \mathrm{P}^{\mathrm{esp}} \\
-\left[0,0, . ., 1_{\mathrm{k}}, \ldots, 0\right] & 0 & \alpha
\end{array}\right]
$$

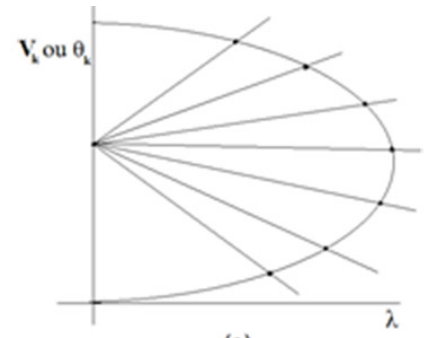

(a)

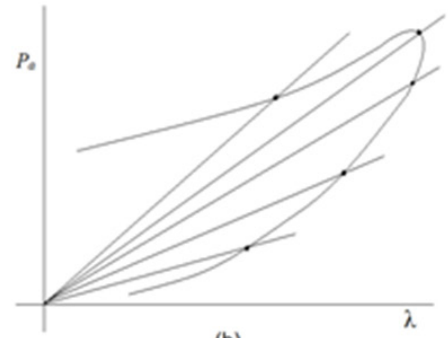

(b)

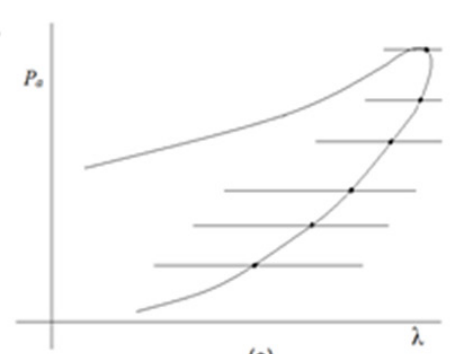

(c)

Figura 2 Métodos: (a) parametrizado por $\alpha$ no plano $\lambda-V_{k}$ ou $\lambda-\theta_{k^{\prime}}$ (b) parametrizado por $\alpha$ no plano $\lambda-P a$, (c) parametrizado por $P a$.

Já no método proposto em Garbelini et al. (2007), que é uma parametrização global, a equação acrescida à Equação (10) é a seguinte:

$$
\mathrm{T}(\theta, \mathrm{V}, \lambda, \alpha)=\alpha\left(\lambda-\lambda^{0}\right)-\left[\mathrm{Pa}(\theta, \mathrm{V})-\mathrm{Pa}^{0}\right]=0
$$

sendo:

$$
P a(\theta, \mathrm{V})=\sum_{\mathrm{i} e \mathrm{j} \in \Omega} \mathrm{g}_{\mathrm{ij}}\left[\mathrm{V}_{\mathrm{i}}^{2}+\mathrm{V}_{\mathrm{j}}^{2}-2 \mathrm{~V}_{\mathrm{i}} \mathrm{V}_{\mathrm{j}} \cos \left(\theta_{\mathrm{i}}-\theta_{\mathrm{j}}\right)\right]
$$

e $\alpha$ o coeficiente angular da reta (parâmetro utilizado). Pa representa a perda total de potência ativa, por isso trata-se de uma técnica de parametrização global, Figura 2(b). Caso $\alpha=0$ e variando-se o valor de $P a^{0}$, a solução do sistema (29) resultará em $\mathrm{Pa}$ como parâmetro (ALVES et al., 2000), Figura 2(c). Linearizando pelos dois primeiros termos da série de Taylor, obtém-se o sistema:

$$
-\left[\begin{array}{ccc}
-\mathbf{J} & & \mathrm{P}^{\text {esp }} \\
\frac{\partial \mathrm{T}}{\partial \theta} & \frac{\partial \mathrm{T}}{\partial \mathrm{V}} & \alpha
\end{array}\right]\left[\begin{array}{c}
\Delta \mathbf{x} \\
\Delta \lambda
\end{array}\right]=-\mathbf{J}_{\mathbf{m}}\left[\begin{array}{c}
\Delta \mathbf{x} \\
\Delta \lambda
\end{array}\right]=\left[\begin{array}{c}
\Delta \mathbf{G} \\
\mathrm{T}
\end{array}\right]
$$

no qual a matriz $\mathrm{J}_{\mathrm{m}}$ é dada por: 


$$
\mathbf{J}_{\mathbf{m}}=\left[\begin{array}{ccc} 
& & \mathrm{P}^{\mathrm{esp}} \\
\frac{\partial \mathrm{T}}{\partial \theta} & \frac{\partial \mathrm{T}}{\partial \mathrm{V}} & \alpha
\end{array}\right]
$$

Por fim, no método proposto em Bonini Neto e Alves (2010 $)$, que também é uma técnica de parametrização global, acrescenta-se ao sistema de Equações (10) a seguinte equação:

$$
S(\theta, V, \lambda, \alpha)=\alpha\left(\lambda-\lambda^{0}\right)-\left(\frac{Y}{n}-\frac{Y^{0}}{n}\right)=0
$$

sendo n o número de barras do sistema e: $Y=\sum_{i=1}^{n} V_{i}$ ou $Y=\sum_{i=1}^{n} \theta_{i}$.

Expandindo em série de Taylor, considerando $Y=\sum_{i=1}^{n} V_{i}$, tem-se o seguinte sistema de equações, que, na forma matricial, torna-se:

$$
-\left[\begin{array}{ccc}
-\mathbf{J} & \mathrm{P}^{\text {esp }} \\
0 & \frac{1}{\mathrm{n}} & \alpha
\end{array}\right]\left[\begin{array}{c}
\Delta \mathbf{x} \\
\Delta \lambda
\end{array}\right]=-\mathbf{J}_{\mathbf{m}}\left[\begin{array}{c}
\Delta \mathbf{x} \\
\Delta \lambda
\end{array}\right]=\left[\begin{array}{c}
\Delta \mathbf{G} \\
\mathrm{S}
\end{array}\right]
$$

A matriz $\mathrm{J}_{\mathrm{m}}$ do método passa a ser dada por:

$$
\mathbf{J}_{\mathbf{m}}=\left[\begin{array}{ccc}
-\mathbf{J} & \mathrm{P}^{\text {esp }} \\
0 & \frac{1}{\mathrm{n}} & \alpha
\end{array}\right]
$$

Já para $Y=\sum_{i=1}^{\mathrm{n}}$, , a matriz $\mathrm{J}_{\mathrm{m}}$ passa a ser dada por:

$$
\mathbf{J}_{\mathbf{m}}=\left[\begin{array}{ccc}
-\mathbf{J} & \mathrm{P}^{\mathrm{esp}} \\
\frac{1}{\mathrm{n}} & 0 & \alpha
\end{array}\right]
$$


A Figura 3(a) apresenta a geometria da trajetória de soluções do método proposto em Bonini Neto e Alves $\left(2010_{\mathrm{b}}\right)$.

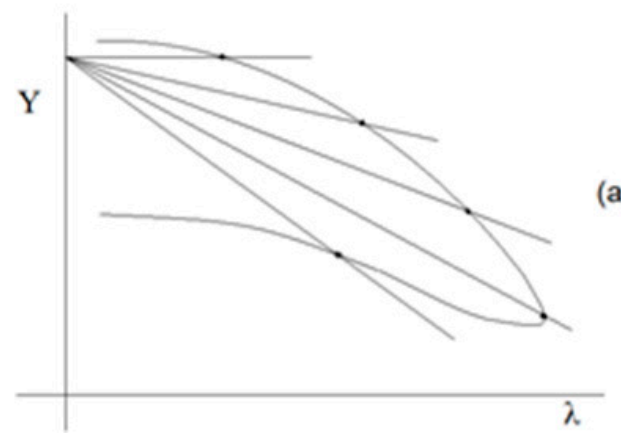

(a)

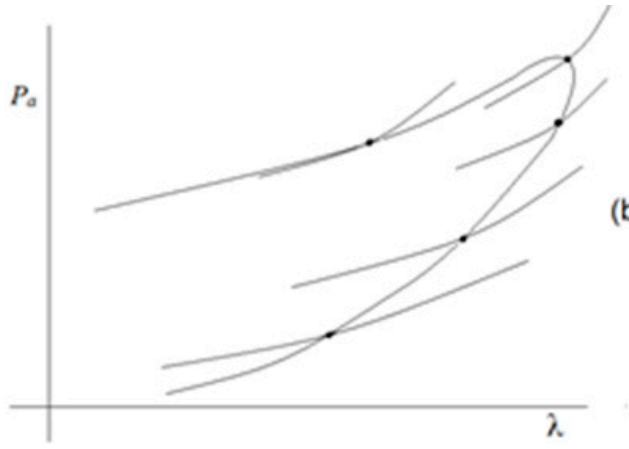

Figura 3 Métodos: (a) parametrizado por $\alpha$ no plano $\lambda-\mathbf{Y}$, (b) parametrizado por parábolas.

Em Magalhães et al. (2013), a equação acrescida à Equação (10) é dada por:

$$
\mathrm{M}(\theta, \mathrm{V}, \lambda, \alpha)=\operatorname{Pa}(\theta, \mathrm{V}, \lambda)-\left[a(\lambda+\alpha)^{2}+b(\lambda+\alpha)+c\right]=0
$$

sendo que $P a$, a perda total de potência ativa, é dada pela Equação (28), enquanto os coeficientes a, b e c são calculados através do desenvolvimento do produtório do coeficiente do polinômio interpolador de Lagrange para três pontos conhecidos do plano $\lambda-P a$. Após a determinação desses coeficientes, para cada valor atribuído ao parâmetro $\alpha$, definem-se novas equações de parábolas, ver Figura $3(b)$. Nesse caso, a matriz $\mathbf{J}_{\mathrm{m}}$ passa a ser dada por:

$$
\mathbf{J}_{\mathbf{m}}=\left[\begin{array}{ccc}
\mathrm{G}, & \mathrm{G}_{\mathrm{V}} & \mathrm{G}_{»} \\
\partial P a / \partial, & \partial P a / \partial \mathrm{V} & -(2 a(\lambda+\alpha)+b)
\end{array}\right]
$$

\section{RESULTADOS}

Neste trabalho, a singularidade da matriz $\mathbf{J}$ é verificada através da inversão do sinal do determinante no PMC, ou seja, o determinante é nulo exatamente no PMC, podendo ser verificado em sistemas de pequeno porte por meio do gráfico dos valores numéricos do determinante em função do carregamento $\lambda$. A dimensão da matriz quadrada J é da ordem de $\left(2 n_{\mathrm{PQ}}+\mathrm{n}_{\mathrm{PV}}\right)$, em que $\mathrm{n}_{\mathrm{PQ}}$ e $\mathrm{n}_{\mathrm{PV}}$ correspondem ao número de barras $\mathrm{PQ}$ e $\mathrm{PV}$, respectivamente. Os resultados obtidos com esses métodos para os sistemas IEEE-14 e 300 barras mostram que as características de convergência dos métodos de fluxo de potência são melhoradas 
na região do PMC, possibilitando a sua determinação com a precisão desejada sem os problemas numéricos relacionados à singularidade da matriz J.

\subsection{Resultados para o Sistema IEEE-14 barras (sub2)}

A Figura 4 apresenta as curvas P-V de algumas barras do sistema estudado, a barra $2(\mathrm{PV})$ e a barra crítica, a barra $14(\mathrm{PQ})$. Os pontos "a" e "b" representam os pontos críticos das curvas que serão analisados. A Figura 5(a) mostra os valores do determinante em torno do ponto "a" (PMC) para ambas as curvas $\left(\mathrm{V}_{2} \mathrm{e}\right.$ $\mathrm{V}_{14}$ ) considerando $\lambda$ como parâmetro (Equação 4 ou 17). Observa-se que o valor do determinante é nulo nesse ponto, comprovando assim a singularidade. Se o parâmetro é a tensão da barra $2\left(\mathrm{~V}_{2}\right)$ (Equação 18, segunda matriz), a singularidade ocorre no ponto "b" (1.5626648, 0.8618405), Figura 5(b). As Tabelas 1 e 2 mostram os valores dos determinantes para ambos os pontos; há uma mudança de sinal de $|\mathrm{J}|$ e $\left|\mathrm{J}_{\mathrm{m}}\right|$ para o fluxo de carga convencional e o fluxo de carga continuado parametrizado por $\lambda$ e por $\mathrm{V}_{2}$.

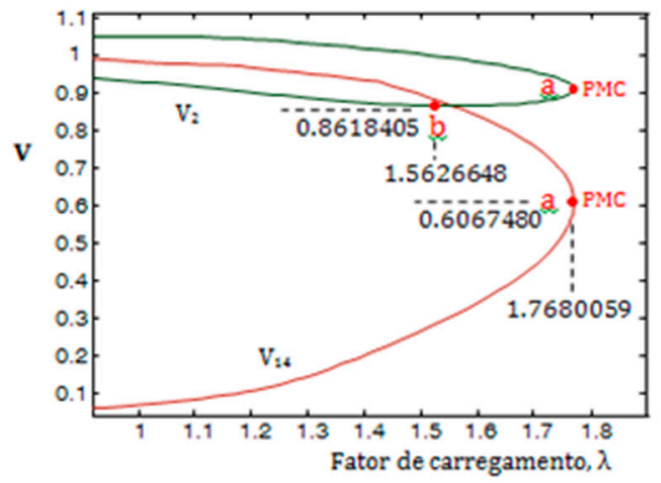

Figura 4 Curva P-V da barra 2 (geração) e barra 14 (crítica).
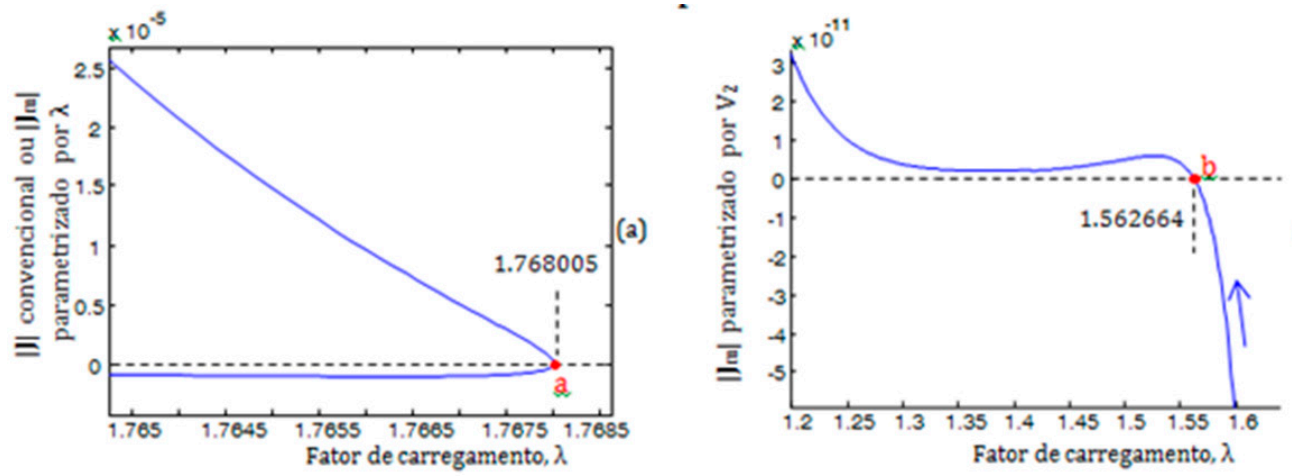

(b)

Figura 5 Determinantes: (a) $|\mathbf{J}|$ e $\left|\mathbf{J}_{\mathbf{m}}\right|$ considerando $\lambda$ como parâmetro, (b) $\left|\mathbf{J}_{\mathbf{m}}\right|$ considerando $V_{2}$ como parâmetro. 
Tabela 1 Ponto " $a$ " considerando $\lambda$ como parâmetro

\begin{tabular}{|c|c|c|c|}
\hline Fator de carregamento, $\lambda$ & $\begin{array}{c}|J| \text { ou }\left|J_{m}\right| \\
\lambda \text { como parâmetro }\end{array}$ & $V_{2}$ & $\mathbf{V}_{14}$ \\
\hline . & .. & ..... & $\ldots \ldots$ \\
\hline 1.767998482 & 0.00000031169 & 0.907351402 & 0.6087480 \\
\hline 1.768005000 & 0.00000017405 & 0.907079398 & 0.6077480 \\
\hline a 1.768005958 & a 0.00000004678 & a 0.906807932 & a 0.6067480 \\
\hline a 1.768001367 & $a-0.00000007069$ & a 0.906537005 & a 0.6057480 \\
\hline 1.767991234 & -0.00000017894 & 0.906266621 & 0.6047480 \\
\hline 1.767975571 & -0.00000027851 & 0.905996782 & 0.6037480 \\
\hline
\end{tabular}

a PMC: singularidade no ponto "a" quando da parametrização por $\lambda$.

Tabela 2 Ponto " $b$ " considerando $\mathrm{V}_{2}$ como parâmetro

\begin{tabular}{ccc}
\hline Fator de carregamento, $\lambda$ & $\mathrm{J}_{\mathrm{m}}, \mathbf{V}_{2}$ como parâmetro & $\mathbf{V}_{2}$ \\
\hline$\ldots \ldots \ldots$ & $\ldots \ldots \ldots$ & $\ldots \ldots \ldots$ \\
1.5652916601094 & -0.000000000001588 & 0.861843210824326 \\
1.5639801355059 & -0.000000000000824 & 0.861841272811978 \\
b 1.5626648654916 & $\mathrm{~b}-0.000000000000117$ & $\mathrm{~b} 0.861840543008172$ \\
b 1.5613458585610 & $\mathrm{~b} 0.000000000000535$ & $\mathrm{~b} 0.861841022032640$ \\
1.5600231233877 & 0.000000000001135 & 0.861842710506932 \\
1.5586966688267 & 0.000000000001687 & 0.861845609055415 \\
$\ldots \ldots \ldots$ & $\ldots \ldots \ldots$ & $\ldots \ldots \ldots$ \\
\hline
\end{tabular}

b: singularidade no ponto " $b$ " quando da parametrização por $\mathrm{V}_{2}$.

A Figura 6(a) mostra a curva $\lambda-\theta_{14}$ e os pontos de bifurcações "a" e "c". Quando da parametrização por $\lambda$, a singularidade ocorre no ponto "a" (observe a mudança de sinal na Tabela 3). Por outro lado, se o parâmetro escolhido for a variável ${ }_{14}$ (Equação 18, primeira matriz), a singularidade não ocorrerá no ponto “a” (Tabela 4), mas sim no ponto "c", ver Figura 6(b) e Tabela 5. 

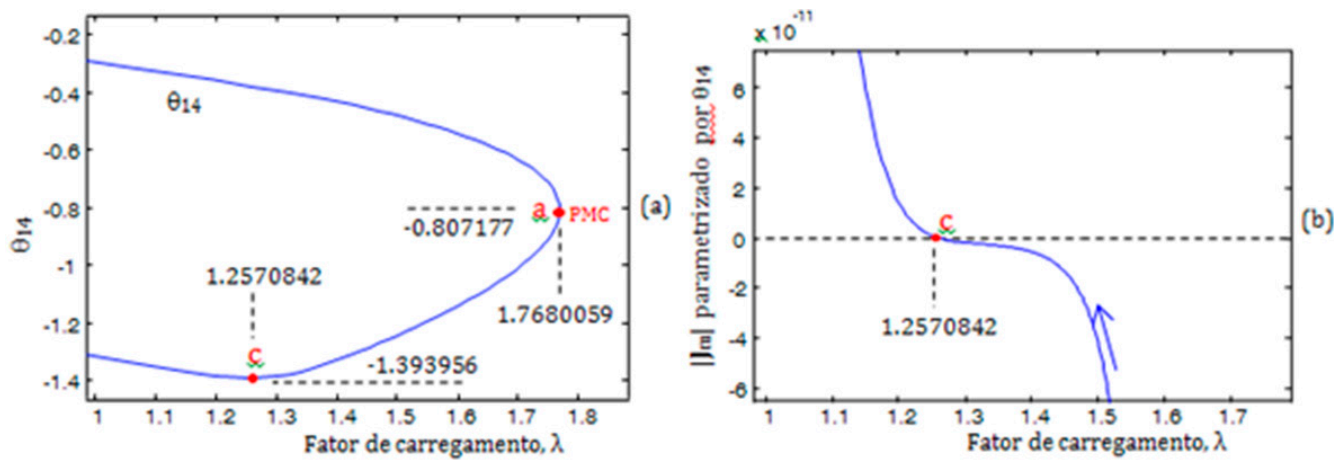

Figura 6 Curva $\lambda-\theta_{14}$ da barra 14 (crítica): (a) pontos de bifurcações, (b) $\left|\mathbf{J}_{\mathbf{m}}\right|$ considerando $\theta_{14}$ como parâmetro.

Tabela 3 Ponto " $a$ " considerando $\lambda$ como parâmetro

\section{Fator de carregamento, $\lambda$}

......

1.7679984824644

1.7680050005793

a 1.7680059589211

a 1.7680013671166

1.7679912347825

1.7679755715262
|J ou $\left|J_{m}\right|, \lambda$ como parâmetro

0.0000003116988

0.0000001740514

a 0.0000000467867

a -0.0000000706901

$-0.0000001789443$

$-0.0000002785122$ $\theta 14$ [radiano]

$-0.80479908404$

$-0.80598784904$

a -0.80717713838

a -0.80836695387

$-0.80955729729$

$-0.81074817044$

a PMC: singularidade no ponto "a" quando da parametrização por $\lambda$.

Tabela 4 Ponto " $a$ " considerando $\theta_{14}$ como parâmetro

Fator de carregamento, $\lambda$

1.7679984824644

1.7680050005793

a 1.7680059589211

1.7680013671166

1.7679912347825

1.7679755715262

\section{$|\mathrm{Jm}|, \theta_{14}$ como parâmetro}

.

$-0.0000499377810$

$-0.0000480284847$

a -0.0000461908827

$-0.0000444223353$

$-0.0000427202979$

$-0.0000410823179$

\section{$\theta_{14}$ [radiano]}

$-0.8047990840469$

$-0.8059878490435$

a -0.8071771383853

$-0.8083669538718$

$-0.8095572972967$

$-0.8107481704477$

a PMC: sem singularidade quando da parametrização por $\theta_{14}$. 
Tabela 5 Ponto "c" considerando $\theta_{14}$ como parâmetro

\begin{tabular}{ccc}
\hline Fator de carregamento, $\lambda$ & $|\mathrm{Jm}|, \theta_{14}$ como parâmetro & $\theta_{14}$ [radiano] \\
\hline$\ldots \ldots \ldots$ & $\ldots \ldots \ldots$ & $\ldots \ldots .$. \\
1.2643710791335 & -0.000000000000569 & -1.3938119425239 \\
1.2619745056267 & -0.0000000000000383 & -1.3938947684508 \\
c 1.2595459734450 & $\mathrm{c}-0.000000000000179$ & $\mathrm{c}-1.3939433274054$ \\
c 1.2570842914437 & $\mathrm{c} 0.000000000000046$ & $\mathrm{c}-1.3939569888814$ \\
1.2545882343822 & 0.000000000000294 & -1.3939351221207 \\
1.2520565425902 & 0.000000000000567 & -1.39387770966884 \\
$\ldots \ldots .$. & $\ldots \ldots \ldots$ & $\ldots \ldots \ldots$ \\
\hline
\end{tabular}

c: singularidade no ponto "c" quando da parametrização por $\theta_{14}$.

A Figura 7 apresenta os valores do $\left|\mathrm{J}_{\mathrm{m}}\right|$ em torno do ponto " $\mathrm{a}$ " (PMC) para o método parametrizado por $\alpha$ (Equação 24) no plano $\lambda-V_{14}$ (Bonini Neto e Alves, 2014 ). $_{\mathrm{b}}$. Observa-se que não há ponto no gráfico que intersecta o eixo das abscissas, no caso $\lambda$, mostrando que não há singularidade da matriz $\mathrm{J}_{\mathrm{m}}$ no ponto crítico "a", quando $\alpha$ é usado como parâmetro, ou seja, não há mudança de sinal de $\mathrm{IJ}_{\mathrm{m}} \mathrm{l}$, Tabela 6.

Outra análise realizada utilizando o método proposto em Bonini Neto e Alves $\left(2014_{b}\right.$ ) foi a parametrização por $\alpha$ (Equação 24 ) no plano $\lambda-V_{2}$. A Figura $8(a)$ mostra a curva com os respectivos pontos de singularidade "a" e "b". O ponto "d" torna-se um ponto de singularidade da matriz $\mathrm{J}_{\mathrm{m}}$ devido à linha " $\mathrm{R}$ " tangenciar a curva $\mathrm{P}-\mathrm{V}$ da barra 2 no ponto "d", o que pode ser confirmado na Figura 8(b) e na Tabela 7.

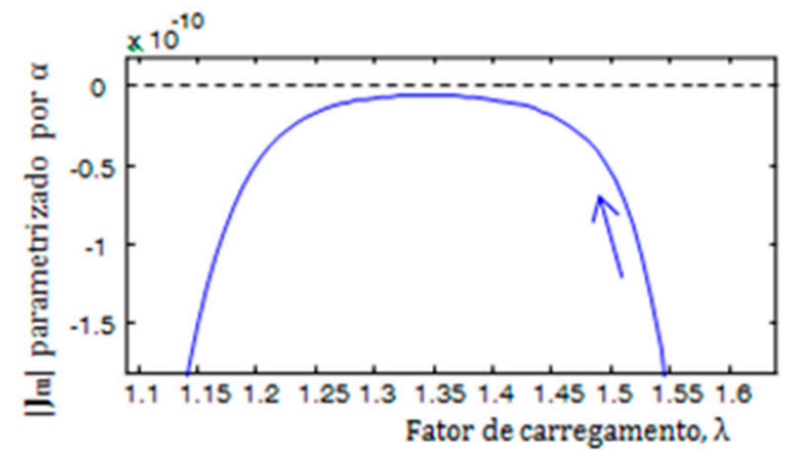

Figura $7\left|\mathrm{~J}_{m}\right|$ considerando $\alpha$ como parâmetro no plano $\lambda-V_{14 .}$

A Figura 9 apresenta a curva $\lambda-\theta_{14}$ da barra crítica com os pontos de singularidade "a" (1.7680005, -46.22935) e "c" (1.257106, -79.876201). Por outro lado, quando da parametrização por $\alpha$ (Equação 26), ambos os pontos não apresentam singularidades, ver Tabelas 8 e 9 . 
Resultados similares são obtidos pelo método proposto em Garbelini et al. (2007). As singularidades são eliminadas para os pontos “a” e "e", conforme mostrado na Figura 10.

Tabela 6 Ponto "a" parametrizado por $\alpha$ no plano $\lambda-V_{14}$

\begin{tabular}{ccc}
\hline Fator de carregamento, $\lambda$ & $|\mathrm{Jm}| .10^{23} \alpha$ como parâmetro & $\mathbf{V}_{14}$ \\
\hline$\ldots$. & $\ldots \ldots$ & $\ldots .$. \\
1.76796996665 & -0.0052014966333 & 0.61083649067 \\
1.76799827999 & -0.0048539091095 & 0.60906706447 \\
a 1.76800914514 & $\mathrm{a}-0.0045293534916$ & $\mathrm{a} 0.60729849650$ \\
1.76800264117 & -0.0042263258334 & 0.60553083488 \\
1.76797884874 & -0.0039434182766 & 0.60376412732 \\
$\ldots \ldots$ & $\ldots \ldots$ & $\ldots .$. \\
\hline
\end{tabular}

a PMC: sem singularidade quando da parametrização por $\alpha$ no plano $\lambda-V_{14}$.
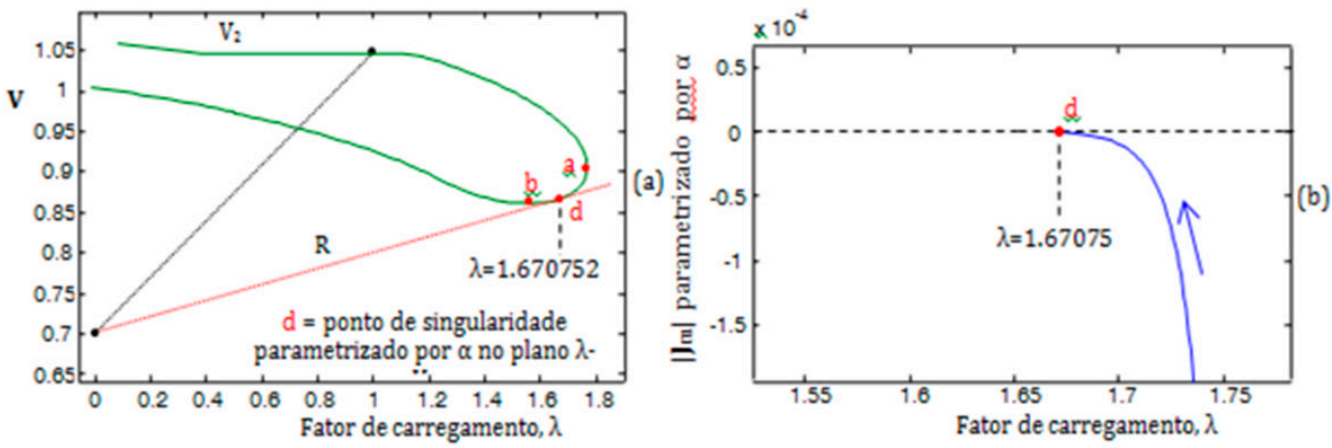

Figura 8 Curva P-V da barra 2 (geração): (a) pontos de bifurcações, (b) $\left|\mathrm{J}_{\mathbf{m}}\right|$ parametrizado por $\alpha$ no plano $\lambda-V_{2}$.

Tabela 7 Ponto "d" parametrizado por $\alpha$ no plano $\lambda-V_{2}$

\begin{tabular}{ccc}
\hline Futor de carregamento, $\lambda$ & $\left.\right|_{\mathrm{m}} \mid \alpha$ como parâmetro & $\mathbf{V}_{2}$ \\
\hline$\ldots$. & $\ldots \ldots$ & $\ldots \ldots$ \\
1.685441767918 & -0.000002860134 & 0.869198448762 \\
1.681649878108 & -0.000002012331 & 0.868649622819 \\
$\mathrm{~d} 1.677009358086$ & $\mathrm{~d}-0.000001260642$ & $\mathrm{~d} 0.868016532528$ \\
$\mathrm{~d} 1.670752186667$ & $\mathrm{~d} 0.000000004573$ & $\mathrm{~d} 0.867222562629$ \\
$\ldots \ldots$ & $\ldots \ldots$ & $\ldots \ldots$ \\
\hline
\end{tabular}

$d$ : singularidade no ponto " $d$ " quando da parametrização por $\alpha$ no plano $\lambda-V_{2}$. 


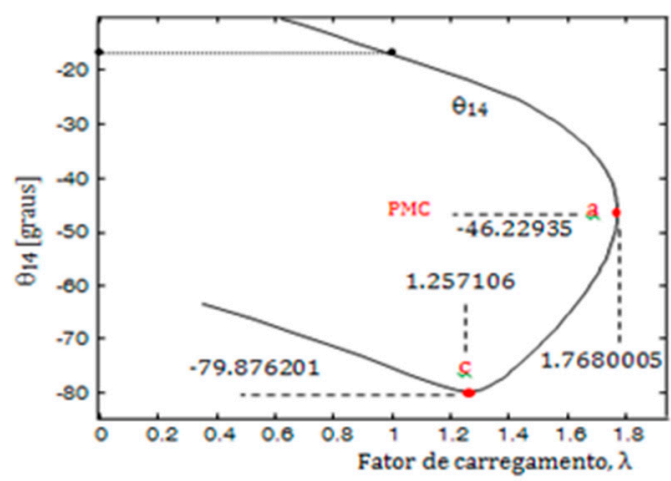

Figura 9 Curva $\lambda-\theta_{14}$ da barra 14 (crítica).

Tabela 8 Ponto " $a$ " parametrizado por $\alpha$ no plano $\lambda-\theta_{14}$

\begin{tabular}{ccc}
\hline Fator de carregamento $\lambda$ & $\left.\right|_{\mathrm{m}} \mid \alpha$ como parâmetro & $\theta_{14}$ [graus] \\
\hline$\ldots$ & $\ldots$ & $\ldots$. \\
1.76736986768 & -0.0000082378026 & -45.2062516798 \\
1.76784682955 & -0.0000062054812 & -45.7203543033 \\
$a 1.76800057754$ & $a-0.00000468447367$ & $\mathrm{a}-46.2293597562$ \\
1.76784762807 & -0.0000035445805 & -46.7332694217 \\
1.76740391545 & -0.0000026880424 & -47.2320936030 \\
$\ldots$ & $\ldots$. & $\ldots$. \\
\hline
\end{tabular}

a PMC (sem singularidade quando da parametrização por $\alpha$ no plano $\left.\lambda-\theta_{14}\right)$.

Tabela 9 Ponto "c" parametrizado por $\alpha$ no plano $\lambda-\theta_{14}$

\begin{tabular}{ccc}
\hline Fator de carregamento $\lambda$ & $\mathrm{J}_{\mathrm{m}} \mid \alpha$ como parâmetro & $\theta_{14}$ [graus] \\
\hline$\ldots$ & $\ldots$ & $\ldots$ \\
1.2709861197 & -0.000000000001170 & -79.84380960672 \\
1.2641715114 & -0.000000000001278 & -79.86823029107 \\
$\mathrm{c} 1.2571066673$ & $\mathrm{c}-0.000000000001411$ & $\mathrm{c}-79.87620121853$ \\
1.2497804440 & -0.000000000001576 & -79.86694547798 \\
1.2421857527 & -0.000000000001780 & -79.83987897687 \\
$\ldots$ & $\ldots$. & $\ldots$ \\
\hline
\end{tabular}

c: sem singularidade quando da parametrização por $\alpha$ no plano $\lambda-\theta_{14}$. 


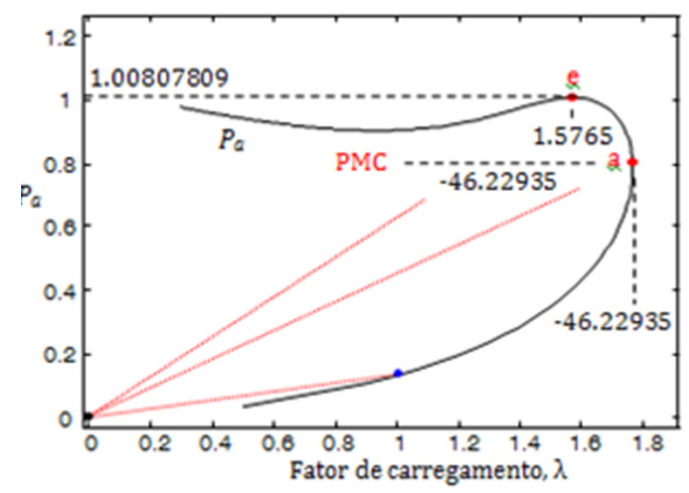

Figura 10 Curva $\lambda-P a$.

A Figura 11 apresenta a curva $\lambda-\Sigma \mathrm{V}$ com os pontos de singularidades, o PMC “a” (1.7680149, 0.7423153) e o "f” (1.356686, 0.531313), pelo método proposto em Bonini Neto e Alves (2010b). Considerando $\alpha$ como parâmetro (Equação 33), ambos os pontos não apresentaram singularidades.

Na Figura 12(a), pode-se ver a curva $\lambda-\Sigma \theta$ com os respectivos pontos de intersecção "a" e " $\mathrm{f}$ ", para os quais também não ocorreram singularidades. Já no ponto "g”, ocorre uma singularidade devido à linha " $\mathrm{R}$ ” tangenciar a curva $\lambda-\Sigma \theta$ neste ponto, causando a singularidade (Equação 34), Figura 12(b), conforme se pode comprovar na Tabela 10 pela mudança de sinal de $\left|\mathrm{J}_{\mathrm{m}}\right|$.

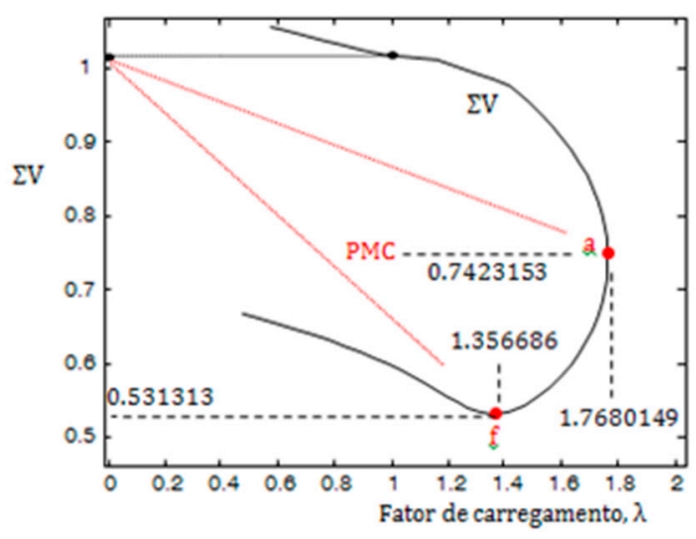

Figura 11 Curva $\lambda-\Sigma V$. 

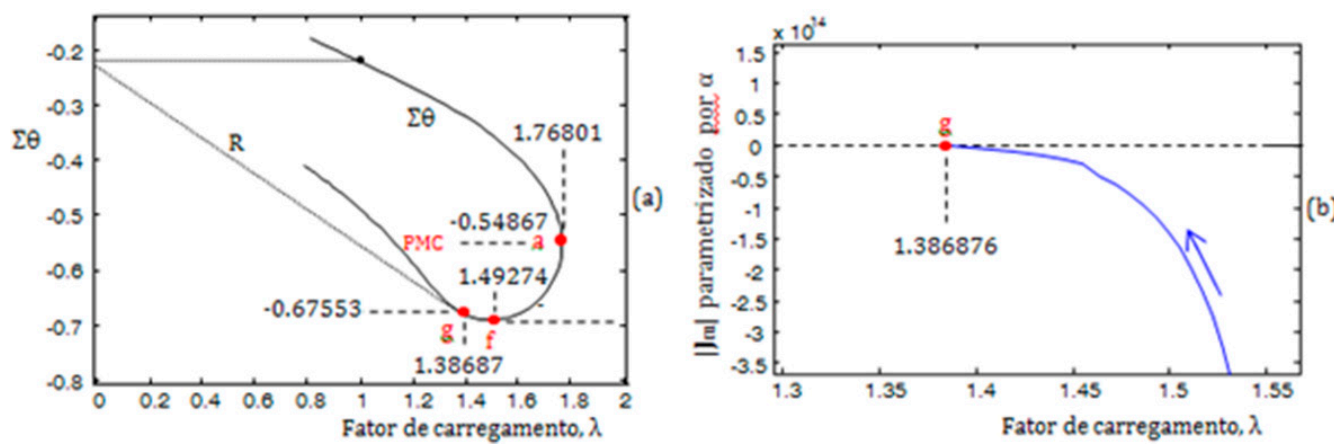

Figura 12 Curva $\lambda-\Sigma \theta$ : (a) pontos singulares (b) $\left|\mathbf{J}_{m}\right|$ parametrizado por $\alpha$ no plano $\lambda-\Sigma \theta$.

Tabela 10 Ponto "g" parametrizado por $\alpha$ no plano $\lambda-\Sigma \theta$

\section{Fator de carregamento $\lambda$} ......

1.44540662350

1.43419900963

g 1.41994544710

g 1.38687674164

\section{J Jm|.1025 a como parâmetro}

......

$-0.000000000002252$

$-0.000000000001562$

g -0.000000000000945

g 0.000000000000122

\section{$\Sigma \theta / \mathbf{n}$}

$-0.68565213378$

$-0.68435167501$

g -0.68227949764

g -0.67553147284

g: singularidade no ponto "g" quando da parametrização por $\alpha$ no plano $\lambda-\Sigma \theta$.

A Figura 13 mostra geometricamente a trajetória de soluções e os pontos de singularidade das respectivas matrizes $\mathrm{J}_{\mathrm{m}}$ do método proposto em Magalhães et al. (2013) (que utiliza parábolas) e das demais técnicas propostas neste capítulo.
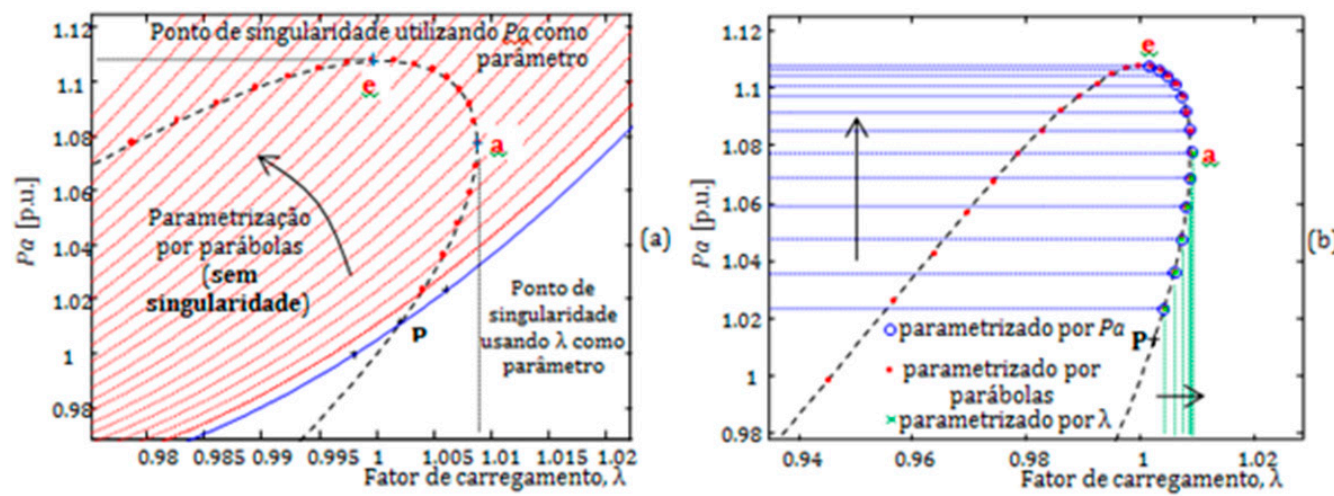

Figura 13 Geometria das soluções dos métodos estudados e respectivos pontos de singularidades na curva $\lambda-P a$ : (a) parametrizado por parábolas, (b) parametrizado por $P a$, parábolas $\mathrm{e} \lambda$. 
Observa-se que o único método que não apresenta problemas numéricos relacionados com a singularidade na obtenção da curva P-V é o que usa a técnica de parametrização por parábolas, ver Figura 13(a). Quando da utilização de $\lambda$ como parâmetro, o método fica restrito pelo ponto "a", enquanto o parametrizado por $\mathrm{Pa}$ fica restrito pelo ponto "e", ver Figura 13(b).

Para o sistema IEEE-14 barras, os pontos de singularidade das matrizes $\mathbf{J}_{\mathrm{m}} \mathrm{e}$ os respectivos valores dos determinantes para cada método é apresentado na Figura 14. Cada ponto de singularidade pode ser observado no gráfico dos valores $\operatorname{dos}\left|J_{\mathrm{m}}\right|$ em função de $\lambda$, ver Figura 14(a) e (b). Nota-se que se $\lambda$ for utilizado como parâmetro da continuação, o método falha na obtenção de pontos da curva $\mathrm{P}-\mathrm{V}$ que se encontram além do PMC, ou seja, fica como visto anteriormente, limitado pelo ponto "a". Isso é comprovado pelo valor do $|\mathrm{J}|$, que se anula no ponto "a", ver Figura 14(a) e (b). No caso dos parâmetros magnitude de tensão da barra 2 $\left(\mathrm{V}_{2}\right)$ e $\mathrm{Pa}$, constata-se que as matrizes Jacobianas são singulares nos respectivos pontos "b", ver Figura 14, e "e", e, como é de se esperar, os seus determinantes se anulam nesses pontos conforme se constata na Figura 14(b). Por outro lado, se a magnitude da tensão da barra crítica $\left(\mathrm{V}_{14}\right)$ ou a variável da equação da parábola localizada no plano $\lambda-P a$ forem utilizadas como parâmetro, as suas matrizes não apresentam singularidades, visto que os seus determinantes não intersectam o eixo da abscissa em nenhum ponto.

Pode-se constatar para o sistema IEEE-14 barras que os gráficos dos determinantes se mantiveram contínuos ao longo de todo o traçado da curva P-V, comprovando que o PMC é consequente de uma bifurcação sela-nó (BSN) relacionada ao limite de capacidade de transmissão de um sistema elétrico, no ponto em que a matriz Jacobiana é singular.

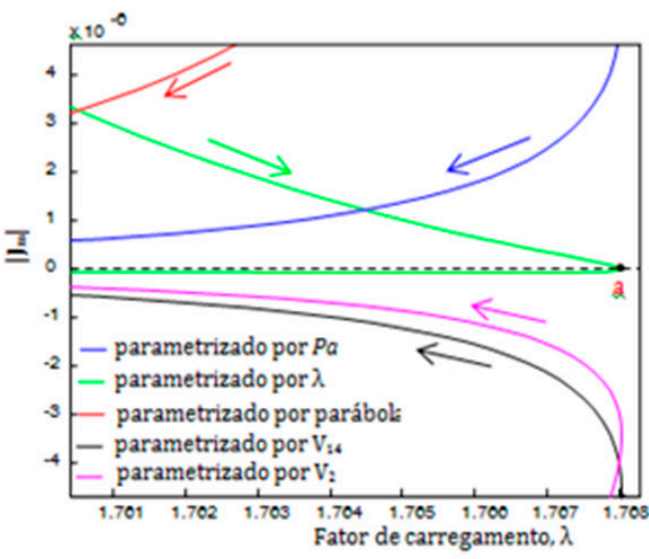

(a)

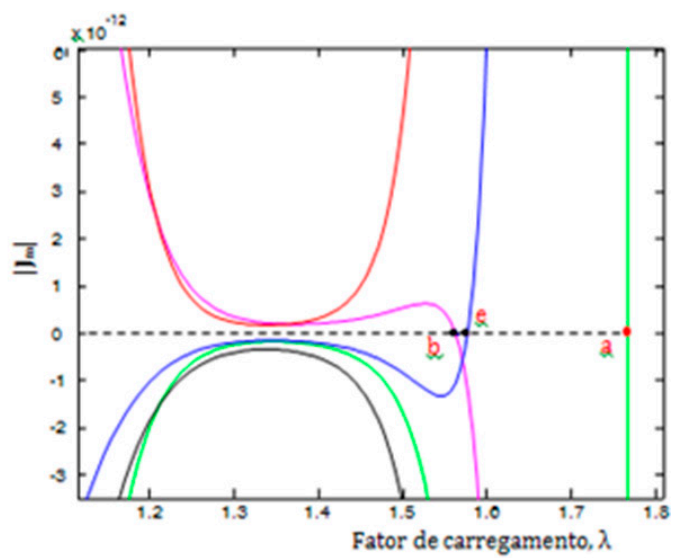

(b)

Figura 14 Pontos de singularidade para o sistema IEEE-14 barras: (a) |J| para cada parâmetro, (b) região ampliada da Figura (a). 


\subsection{Resultados para o Sistema IEEE-300 barras}

O PMC pode ser consequente ou de uma bifurcação sela-nó (BSN), relacionada ao limite de capacidade de transmissão de um sistema elétrico, ou de uma bifurcação estática induzida por limites (BIL), a qual está relacionada com o limite de potência reativa do gerador. Uma simples diferenciação entre as duas pode ser efetuada pela análise do determinante da matriz J, que é singular no caso da BSN, e não singular no caso da BIL. Entretanto, nas diversas condições de carregamento analisadas, os valores numéricos do determinante do sistema IEEE-300 são muito elevados, maiores do que $1 \mathrm{e}+309$, e a sua representação no software MATLAB é efetuada pelo símbolo inf ( $\infty$, infinito), impossibilitando, assim, a sua visualização gráfica. Uma alternativa para possibilitar a visualização gráfica dos valores de seu determinante em função do carregamento $\lambda$ é a sua redução através da divisão dos elementos da matriz por 10. Um estudo no PMC é efetuado com o intuito de verificar se o ponto corresponde a uma BSN ou a uma BIL.

A Figura 15 apresenta os resultados para o sistema teste IEEE de 300 barras. O sistema apresenta 231 barras de carga (PQ) e 68 barras de geração (PV), no entanto, a matriz Jacobiana para esse sistema é da ordem $530 \times 530$, acarretando valores numéricos muito altos para o determinante. Nas figuras, são mostradas duas curvas em função da mudança do tipo da barra de geração $\left(\mathrm{V}_{7062}\right)$ de PV para PQ, a qual ocorre muito próxima ao PMC. Essa mudança acontece em função de o limite máximo de geração de potência reativa $\left(\mathrm{Qg}_{\max }=1,5\right.$ p.u. de MVAr) ter sido atingido. As curvas 1 e 2 são utilizadas para avaliar se o PMC é causado por uma BSN ou uma BIL. A curva 1 é obtida utilizando os dados originais do banco de dados do sistema, enquanto a curva 2 é traçada considerando-se a barra de geração 7062 (originalmente do tipo PV) como sendo uma barra de carga (tipo $\mathrm{PQ})$ com a sua potência reativa gerada fixada no seu valor máximo $\left(\mathrm{Qg}_{7062}=1,5\right.$ p.u. $\left.=\mathrm{Qg}_{\max (7062)}\right)$.

As correspondentes curvas P-V (curva 1 e 2 ) da barra crítica $\left(V_{526}\right)$ são apresentadas na Figura 15(a), enquanto as da barra $V_{7062}$, na Figura 15(b). Na Figura 15(c), são apresentadas as correspondentes curvas 1 e 2 da perda total de potência ativa $(\mathrm{Pa})$ como função de 1 . Nessas figuras, também se pode ver as regiões ampliadas das vizinhanças do PMC.

Observe nesses detalhes que o encontro entre as duas curvas 1 e 2 se dá na parte de baixo da curva P-V, próximo ao PMC. Na Figura 16, apresentam-se, para cada método, os valores numéricos dos determinantes para os casos das curvas 1 e 2 . Observa-se que, no caso da curva 1 , o gráfico dos determinantes apresenta uma descontinuidade próximo ao PMC, mostrando que nesse caso o PMC é ocasionado por uma BIL, ou seja, uma bifurcação induzida por limites (no caso pelo limite de potência reativa do gerador 7062). Observe que se o seu valor 
for fixado em seu valor máximo desde o início do traçado da curva, o PMC seria ocasionado por uma BSN conforme se pode confirmar nas figuras.

$\mathrm{Na}$ Figura 16(a), são apresentadas as duas curvas de valores dos determinantes da matriz Jacobiana $\mathbf{J}$ (Equação 2) do método parametrizado por $\lambda$. A curva 2 comprova que a singularidade do ponto $S_{1}$ corresponde a uma BSN. Como se pode ver, no caso da curva 1 ocorreu uma descontinuidade na curva dos determinantes, a qual, conforme se constata nas regiões ampliadas das Figuras 15(a) e (b), acontece na porção correspondente à parte de baixo da curva P-V. Entretanto, da curva 1 apresentada na Figura 15(a), comprova-se que a singularidade do novo ponto $S_{1}$ também é ocasionada por uma BSN e não por uma BIL.
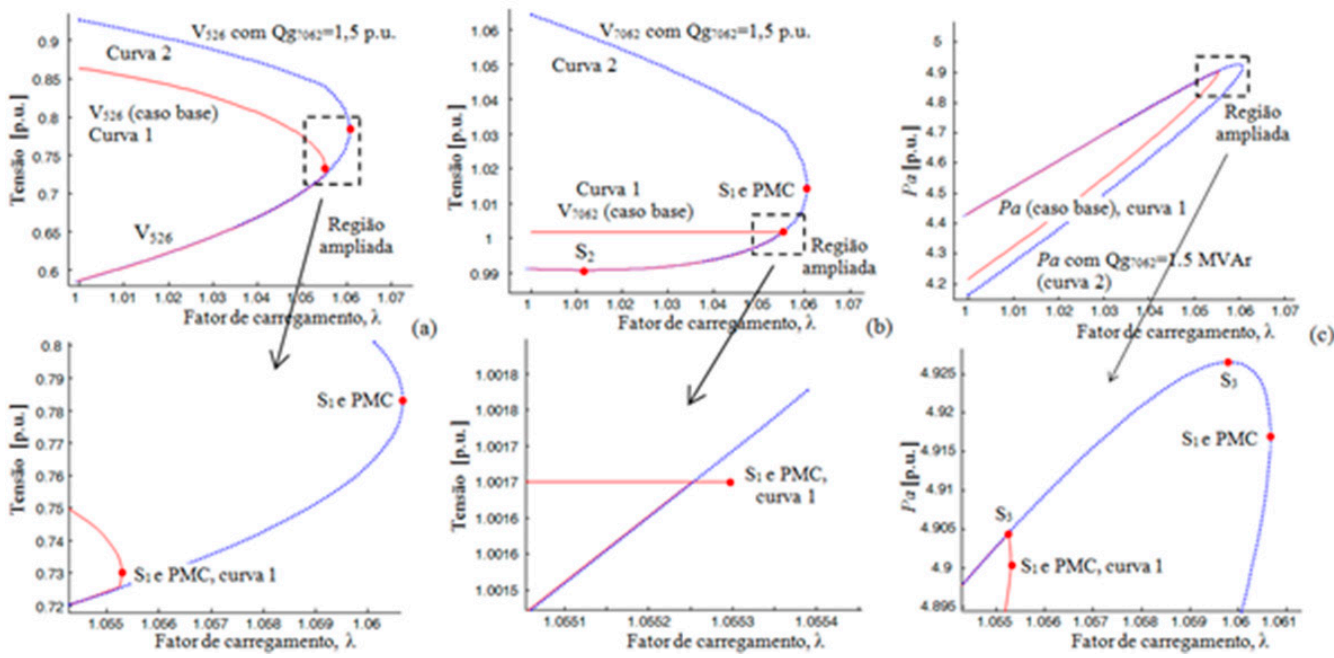

Figura 15 Pontos de singularidade para 0 sistema IEEE-300 barras: (a) curvas $\mathrm{P}-\mathrm{V}_{526}$ para 0 caso base e $\mathrm{P}-\mathrm{V}_{526}$ para $\mathrm{Qg}_{7062}=1,5$ p.u. MVAr, (b) curvas P-V $\mathrm{V}_{7062^{\prime}}$ (c) curva $\lambda$-Pa.

Conforme apresentado na Figura 16(b), o método não apresenta problemas numéricos relacionados com a presença de singularidade se o parâmetro usado for magnitude de tensão da barra $526\left(\mathrm{~V}_{526}\right)$, Equação (3). Já no caso da parametrização por $\mathrm{V}_{7062}$, a singularidade ocorre apenas no ponto $\mathrm{S}_{2}$, ver o detalhe da Figura 16(c). Na Figura 17, são apresentadas as curvas dos valores numéricos dos determinantes das matrizes Jacobianas $\left(\mathbf{J}_{\mathrm{m}}\right)$ dos métodos parametrizados por a (parábolas, Equação 5) e por $\mathrm{Pa}$ (Equação 4). Observe na Figura 17(a) que no caso da utilização de a como parâmetro, ou seja, das equações das parábolas, não há singularidade da matriz Jacobiana em nenhuma das curvas. No entanto, quando da parametrização por $\mathrm{Pa}$, a singularidade ocorre no ponto $\mathrm{S}_{3}$ da curva 2, ver Figura 17(b). Observa-se na curva 1 da Figura 17(b) que o gráfico do determinante da matriz Jacobiana, $|\mathrm{J}|$, apresenta um salto exatamente no ponto $S_{3}$, 
no entanto pode se constatar que o método utilizando $P a$ também identifica esse ponto como sendo ocasionado por uma SNB, ver Figura 17(c). Observe na Figura $17(\mathrm{c})$ que o fator de carregamento em $\mathrm{S}_{1}$ é ligeiramente maior que o de $\mathrm{S}_{3}$.
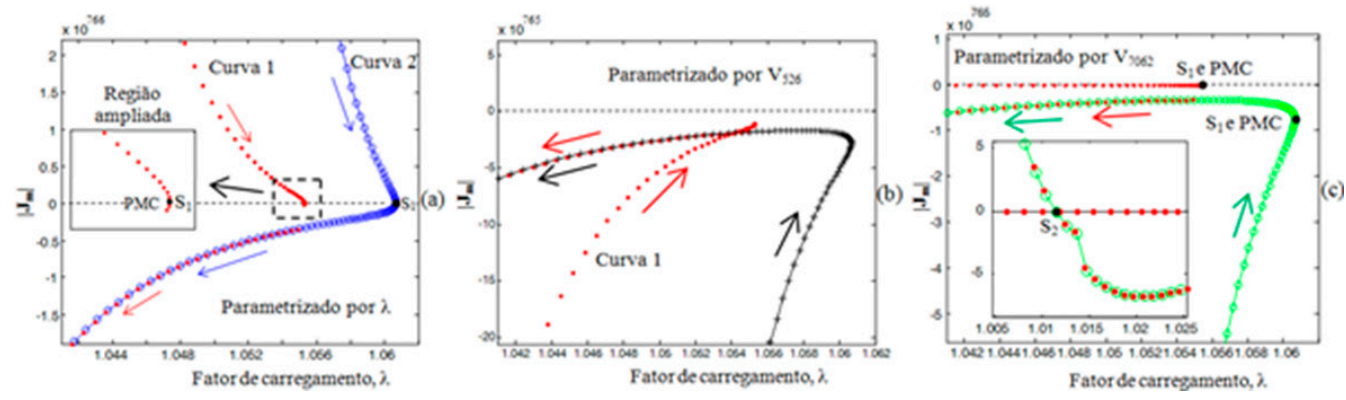

Figura $16|\mathrm{~J}|:$ : (a) parametrizado por $\mathrm{I}$ (b) parametrizado por $\mathrm{V}_{56^{\prime}}$ (c) parametrizado por $\mathrm{V}_{7062}$.
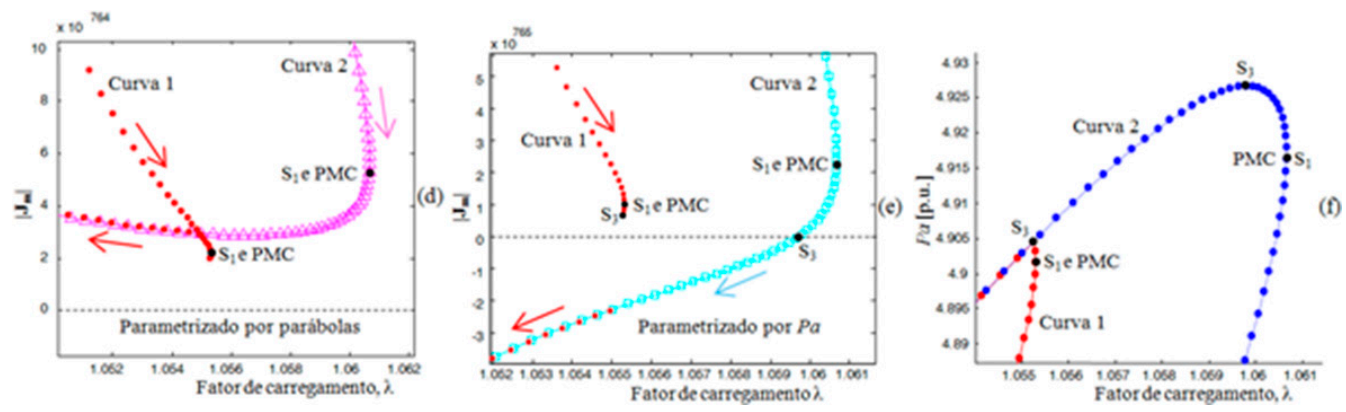

Figura $17|\mathrm{~J}|$ : (a) parametrizado por parábolas, (b) parametrizado por $P a,(c)$ curvas $\lambda$ - $P a$.

\section{CONCLUSÕES}

Neste capítulo, foi apresentada uma análise da singularidade da matriz Jacobiana do fluxo de carga continuado para várias técnicas de parametrização geométrica visando a obtenção do PMC de sistemas elétricos de potência. Os parâmetros utilizados foram: o fator de carregamento $(\lambda)$, a magnitude de tensão da barra crítica ou de uma barra específica, a perda total de potência ativa $(P a)$, o coeficiente angular da reta e equações de parábolas.

O ponto de máximo carregamento pode ser consequente ou de uma bifurcação de sela-nó (BSN) relacionada ao limite físico de capacidade de transmissão num sistema elétrico, onde a matriz Jacobiana é singular, ou de uma bifurcação estática induzida por limites (BIL), relacionada com o limite de potência reativa do gerador, onde a matriz não é singular. Uma alternativa para viabilizar a representação gráfica dos valores do determinante em função do carregamento $\lambda$ 
também foi apresentada, que é a redução dos seus valores através da divisão dos elementos da matriz por 10. Com base nessa representação gráfica, neste capítulo, também se apresentou a análise de algumas das várias propostas utilizadas para a melhoria do desempenho do fluxo de carga continuado, visando a obtenção do ponto de máximo carregamento de sistemas elétricos de potência sem os problemas numéricos relacionados à singularidade da matriz $\mathbf{J}$.

\section{REFERÊNCIAS}

AJJARAPU, V.; CHRISTY, C. The continuation power flow: a tool for steady state voltage stability analysis. IEEE Trans. on Power Systems, v. 7, n. 1, p. 416-423, 1992.

ALVES, D. A. et al. Continuation load flow method parameterized by power losses. Proceedings of the Ieee Power Engineering Society Winter Meeting, v. 2, p. 1123-1128, 2000.

BONINI NETO, A.; ALVES, D. A. An improved parameterization technique for the Continuation Power Flow. In: Transmission and Distribution Conference and Exposition, Ieee Pes, New Orleans, 2010a. p. 1-6.

Improved geometric parameterization techniques for continuation power flow.

IET Generation, Transmission \& Distribution, v. 4, p. 1349-1359, 2010 b.

. Studies of contingencies in power systems through a geometric parameterization technique. Part I: mathematical modeling. World Journal Control Science and Engineering, v. 2, n. 1, p. 18-24, 2014a.

. Studies of contingencies in power systems through a geometric parameterization technique. Part II: performance evaluation. World Journal Control Science and Engineering, v. 2, n. 1, p. 25-34, 2014b.

CHIANG, H. D. et al. CPFLOW: a practical tool for tracing power system steady-state stationary behavior due to load and generation variations. IEEE Transactions on Power Systems, v. 10, p. 623-634, 1995.

. The generation of ZIP-V curves for tracing power system steady state stationary behavior due to load and generation variations. IEEE Pes Summer Meeting, Edmonton, p. 647-651, 1999.

GARBELINI, E. et al. An efficient geometric parameterization technique for the continuation power flow. Electric Power Systems Research, v. 77, p. 71-82, 2007.

LI, S. H.; CHIANG, H. D. Nonlinear predictors and hybrid corrector for fast continuation power flow. IET Generation, Transmission \& Distribution, v. 2, p. 341-354, 2008.

MAGALHÃES, E. M.; BONINI NETO, A.; ALVES, D. A. A parameterization technique for the continuation power flow developed from the analysis of power flow curves. Mathematical Problems in Engineering, v. 2012, p. 1-24, 2012.

. Técnica de parametrização geométrica para o fluxo de carga continuado baseado nas variáveis fator de carregamento e perda total de potência ativa. In: Encontro Regional Ibero-Americano do Cigré - XV Eriac, Foz do Iguaçu, p. 1-8, 2013. 
MORI, H.; SEKI, K. Non-linear-predictor-based continuation power flow for unbalanced distribution systems. In: Transmission \& Distribution Conference \& Exposition: Asia and Pacific [s.n], p. 1-4, 26-30, Oct., 2009.

SEYDEL, R. From equilibrium to Chaos: practical bifurcation and stability analysis. 2 . ed. New York: Springer-Verlag, 1994.

ZHAO, J.; ZHANG B. Reasons and countermeasures for computation failures of continuation power flow. In: Proceedings of the Ieee Power Engineering Society General Meeting (Pes '06), June, 2006. 